\title{
VARIÁVEIS FÍSICAS DE ARGISSOLO AMARELO DO SEMIÁRIDO NORDESTINO EM FUNÇÃO DAS OPERAÇÕES DE PREPARO PERIÓDICO
}

\author{
Jardênia Rodrigues Feitosa ${ }^{1}$, Nelci Olszevki ${ }^{2}$, Jorge Wilson Cortez ${ }^{3}$, Hideo de Jesus Nagahama ${ }^{4}$
}

\begin{abstract}
RESUMO
A realização de operações de preparo afeta as propriedades físicas do solo, podendo levar com o tempo a uma redução da sua qualidade. Parâmetros relacionados à estrutura do solo tais como agregação, densidade e porosidade, são comumente utilizados para inferir sobre a qualidade física dos solos agrícolas. Assim, objetivou-se avaliar o estado de agregação, o índice de floculação, a densidade e a porosidade total de Argissolo Amarelo do semiárido nordestino em função dos sistemas de preparo e do número de operações. O experimento foi desenvolvido em área pertencente à Universidade Federal do Vale do São Francisco - UNIVASF, sendo os tratamentos compostos pela combinação de diferentes tipos de preparo e intensidades de operação, os quais foram, respectivamente: sem preparo, preparo com escarificador, com grade leve em "tandem", grade leve "off-set" (discos de 0,56 m de diâmetro) e, grade leve "off-set" (discos de 0,61 m de diâmetro); uma, duas e três operações. Utilizou-se o delineamento em blocos casualizados com quatro repetições, com os tratamentos dispostos em esquema de parcelas subdivididas, sendo os sistemas de preparo dispostos nas parcelas e o número de operação nas subparcelas. Foram coletadas amostras deformadas e indeformadas de solo para avaliação da estabilidade dos agregados do solo, densidade do solo, porosidade total e índice de floculação. Não foram obtidas diferenças significativas para os parâmetros Diâmetro Médio Geométrico (DMG), Diâmetro Médio Ponderado (DMP) e Índice de Estabilidade de Agregados (IEA) quando comparados os sistemas de preparo utilizados. A densidade e a porosidade total do solo também não foram significativamente afetadas pelos sistemas de preparo e o número de operações.
\end{abstract}

Palavras-chave: agregados do solo, preparo do solo, qualidade do solo

\section{ABSTRACT \\ PHYSICAL ATTRIBUTES OF YELLOW ULTISOL OF THE SEMIARID NORTHEASTERN IN FUNCTION OF PERIODIC TILLAGE OPERATIONS}

Preparation procedures affect the physical properties of soil, which over time can lead to a reduction in its quality. Parameters related to soil structure such as state of aggregation, bulk density and porosity are commonly used to infer the physical quality of agricultural soils. Thus, this study aimed to assess the state of aggregation, the flocculation index, bulk density and porosity of Yellow Ultisol in Brazil's northeastern semiarid region in function of tillage systems and the frequency of operations. The experiment was conducted in an area of the Universidade Federal do Vale do São Francisco - UNIVASF, and treatments consisted of combinations of different types and intensities of tillage operations, which were: no tillage, chisel plow, tandem disc harrow, offset disc harrow with discs of $0.56 \mathrm{~m}$ and offset disc harrow with discs of $0.61 \mathrm{~m}$; which received different number of operations (one, two and three). A randomized block design was used with four replications, with treatments arranged in subdivided plots, where the tillage systems make up the plots and number of operations are the subplots. Tilled and undisturbed soil samples were collected to evaluate the stability of soil aggregates, bulk density, porosity and flocculation index. No significant differences were obtained for the parameters Geometric Mean Diameter, mean weighted diameter and aggregate stability index when comparing the tillage systems used. The bulk density and porosity of the soil were not significantly affected by tillage systems or the number of operations.

Keywords: soil aggregates, tillage, soil quality

\section{Recebido para publicação em 24/05/2013. Aprovado em 12/08/2013.}

1 - Eng. Agrícola e Ambiental, Mestranda em Engenharia Agrícola, DEA/UFV, Viçosa, MG. jardenia.feitosa@ufv.br

2 - Engenheira Agrônoma, Profa. Adjunto, Univ. Fed. do Vale do São Francisco, UNIVASF, Juazeiro, BA. nelci.olszevski@univasf.edu.br

3 - Engenheiro Agrônomo, Prof ${ }^{\circ}$ Adjunto, Univ. Fed. da Grande Dourados, FCA/UFGD, Dourados, MS. jorge.cortez@yahoo.com.br

4 - Engenheiro Agrônomo, UNIVASF, Juazeiro, BA. hideo.nagahama@univasf.edu.br

456 REVENG

456-464p. ENGENHARIA NA AGRICULTURA, VIÇOSA - MG, V.21 N.5, SETEMBRO / OUTUBRO 2013 


\section{INTRODUÇÃOO}

A modernização da agricultura brasileira ocasionou transformações nos sistemas de cultivo, destacando-se o uso cada vez maior de máquinas e implementos na condução das operações agrícolas, o que permitiu o seu aprimoramento. No entanto, quando realizado de forma intensiva e em condições inadequadas, o cultivo mecanizado ocasiona modificações nas propriedades físicas do solo, que podem alterar sua qualidade e prejudicar o desenvolvimento das culturas.

Uma das características utilizadas para determinar a qualidade física do solo é a sua estrutura, cuja avaliação pode ser feita por meio de determinações indiretas (quantidade de agregados estáveis em água ou a seco), ou por determinações diretas, por meio de atributos como a densidade do solo, porosidade, índices de floculação e infiltração de água no solo (MENDES et al., 2006). Do ponto de vista agrícola, a estrutura é considerada uma das características mais importantes por ser fundamental no estabelecimento das relações soloplanta. De maneira geral, quanto mais bem agregado for o solo, melhor é a distribuição de poros no perfil, e, consequentemente, melhor é a percolação de água e as trocas gasosas (PEDROTTI; MELLO JÚNIOR, 2009).

Segundo Kemper e Chepil (1965), para expressar o estado de agregação e o tamanho dos agregados do solo, podem ser utilizados parâmetros como o diâmetro médio ponderado (DMP), o diâmetro médio geométrico (DMG) e o índice de estabilidade de agregados (IEA). O DMP demonstra a estabilidade da estrutura frente à ação de desagregação da água, podendo indicar o grau de susceptibilidade do solo à erosão hídrica, apresentando maior valor, quanto maior for a percentagem de agregados grandes (CASTRO FILHO et al., 1998; BERTOL et al., 2004). O DMG representa uma estimativa do tamanho dos agregados de maior ocorrência. Já o IEA denota uma medida da agregação total do solo e não considera a distribuição por classes de agregados.

A densidade é considerada a propriedade física mais dinâmica, uma vez que pode ser alterada pelo cultivo e pela ação de máquinas agrícolas, entre outros fatores (ARAÚJO et al., 2012). Densidade do solo e porosidade total estão entre os principais indicadores físicos da qualidade do solo, por se tratar de características dinâmicas e de fácil determinação, relacionando-se com a compactação do solo e com o crescimento radicular (ARAÚJO et al., 2012). O índice de floculação, que por sua vez representa a proporção de argila naturalmente floculada em relação à argila total, também permite obter respostas sobre o processo de estruturação do solo.

De acordo com Tormena et al. (2002), os sistemas de preparo devem oferecer condições favoráveis ao crescimento e desenvolvimento das culturas. No entanto, a depender do solo, do clima e da cultura, podem promover a degradação da qualidade física do solo. Avaliando as propriedades físicas de um Latossolo submetido a diferentes sistemas de preparo (plantio direto, preparo mínimo e preparo convencional), Tormena et al. (2002) observaram que os mesmos afetaram a densidade do solo entre $0,0-0,10 \mathrm{~m}$ de profundidade, sendo os menores valores encontrados quando realizado o preparo convencional. Observaram ainda que, exceto para o plantio direto, os valores de densidade entre 0,10-0,20 m foram superiores aos encontrados nos primeiros $0,10 \mathrm{~m}$ de profundidade, evidenciando os efeitos dos implementos agrícolas no fracionamento dos agregados nesta camada. Mazurana et al. (2011), avaliando os efeitos de diferentes sistemas de preparo sobre características físicas de um Argissolo Vermelho-Amarelo, observaram que os mesmos interferiram na densidade, porosidade e resistência mecânica à penetração. Quanto à densidade, por exemplo, os autores encontraram os maiores valores nos sistemas de preparo de solo onde o revolvimento foi menor.

Dessa forma, o objetivo desse trabalho foi avaliar o estado de agregação, o índice de floculação, a densidade e a porosidade total de Argissolo Amarelo do semiárido nordestino em função dos sistemas de preparo e do número de operações.

\section{MATERIAL E MÉTODOS}

O experimento foi instalado em área pertencente ao Campus de Ciências Agrárias da Universidade Federal do Vale do São Francisco-UNIVASF, com 
coordenadas geográficas $09^{\circ} 23^{\prime}$ sul e $40^{\circ} 30^{\prime}$ oeste, a uma altitude de $376 \mathrm{~m}$, consistindo na realização de operações de preparo do solo com diferentes números de operações em Argissolo Amarelo distrófico típico de textura arenosa (AMARAL et al., 2006). De acordo com a classificação de Köppen, o clima da região é o tropical semiárido, tipo BSw, caracterizando-se pela escassez e irregularidade da precipitação pluviométrica.

Os tratamentos foram compostos pelas combinações dos sistemas de preparo e o número de operações, os quais foram respectivamente: sem preparo; preparo com escarificador; com grade leve em "tandem"; grade leve "off-set" (discos de 0,56 m ou22" de diâmetro) e, grade leve "off-set" (discos de 0,61 m ou24" de diâmetro); e três quantidades de operações correspondentes, com uma, duas e três passadas do conjunto mecanizado. Como fonte de potência para tracionar os equipamentos de preparo do solo, foi utilizado um trator (tração dianteira auxiliar) com 55,2 kW (75 cv) de potência nominal no motor.

Os tratamentos foram dispostos em esquema de parcelas subdivididas, sendo os sistemas de preparo dispostos nas parcelas e o número de operações nas subparcelas, utilizando-se o delineamento em blocos casualizados com quatro repetições. Cada parcela ocupou uma área de $200 \mathrm{~m}^{2}(10 \mathrm{~m}$ x $20 \mathrm{~m}) \mathrm{e}$ foi subdividida em três subparcelas (3,3 $\mathrm{m} \times 20 \mathrm{~m})$, reservando-se um espaço de $15 \mathrm{~m}$ entre as mesmas para a realização de manobras e estabilização dos conjuntos.

Para avaliar as modificações ocasionadas pelos sistemas de preparo foram coletadas em um ponto dentro de cada subparcela, amostras deformadas e indeformadas de solo nas profundidades de $0,00-0,10 ; 0,10-0,20$ e $0,20-0,30 \mathrm{~m}$, totalizando 180 amostras. As amostras indeformadas foram destinadas a avaliação da estabilidade dos agregados do solo (KIEHL, 1979) com posterior cálculo do diâmetro médio geométrico (DMG) (MELO et al., 2008), diâmetro médio ponderado (DMP) (KIEHL, 1979; CASTRO FILHO et al., 1998) e índice de estabilidade de agregados (IEA) (MORAES et al., 2009). As amostras deformadas destinaram-se a determinação da composição granulométrica do solo e do teor de argila dispersa em água, com posterior cálculo do índice de floculação; e densidade do solo e de partículas, determinadas respectivamente pelo método da proveta e do balão volumétrico (EMBRAPA, 1997), com posterior cálculo da porosidade total (EMBRAPA, 1997).

Os valores dos atributos físicos do solo foram submetidos à análise de variância e teste de Tukey, ao nível de $5 \%$ de probabilidade, utilizando-se o software Assistat 7.6 (SILVA; AZEVEDO, 2002).

\section{RESULTADOS E DISCUSSÃO}

Para o diâmetro médio geométrico (DMG) dos agregados do solo, observou-se que não ocorreram diferenças significativas entre os sistemas de preparo do solo (Figura 1). Comparando-se as médias de DMG para as três profundidades estudadas, os menores valores foram encontrados entre $0,00-0,10 \mathrm{~m}$. O preparo com a grade off-set de discos de 0,61 m resultou na maior discrepância entre os valores de DMG encontrados para as três profundidades, sendo que o diâmetro dos agregados na camada de $0,20-0,30 \mathrm{~m}$ foi maior que o daqueles encontrados entre $0,00-0,10 \mathrm{~m}$, cerca de $0,095 \mathrm{~mm}$.

Resultados semelhantes foram encontrados para os valores de diâmetro médio ponderado (DMP), obtendo-se diferenças significativas apenas quando avaliadas as três profundidades de coleta, sendo os menores valores encontrados entre $0,00-0,10 \mathrm{~m}$ (Figura 1).

Silva e Mielniczuk (1998), ao avaliarem Argissolo Vermelho escuro, observaram que os agregados quando submetidos ao peneiramento por via úmida, apresentavam grandes variações no diâmetro médio ponderado entre os tratamentos e profundidades, sendo os menores valores encontrados nas áreas submetidas à mecanização agrícola. Neste trabalho, no entanto, a área não preparada mecanicamente não apresentou valores de DMG e DMP significativamente superiores às demais.

Os menores valores de DMG eDMP, encontrados entre $0,00-0,10 \mathrm{~m}$, podem ser atribuídos a maior quebra dos agregados na superfície do solo durante o preparo devido ao revolvimento superficial. Além disso, o rompimento dos agregados expõe a matéria orgânica existente em seu interior, o que 

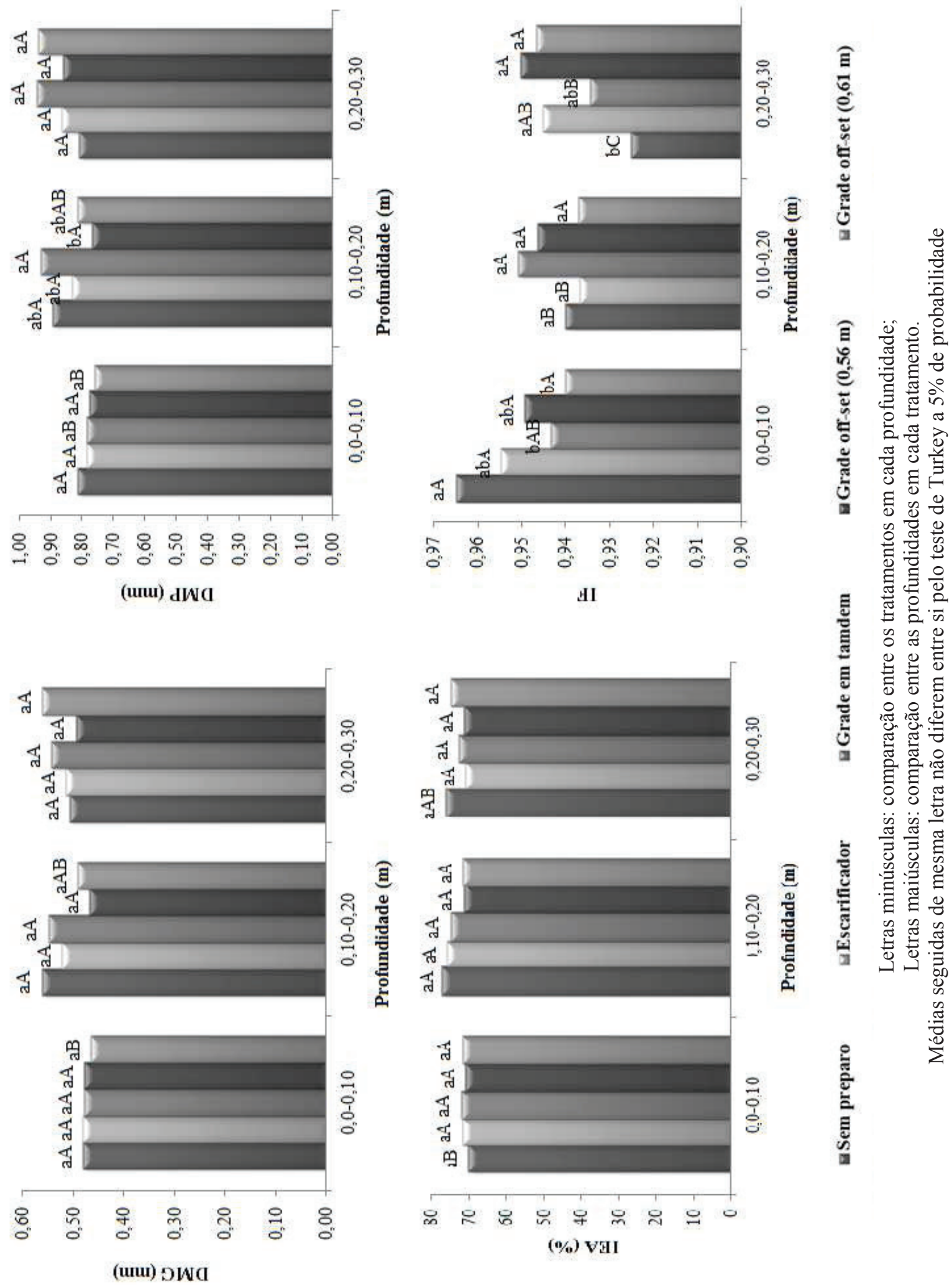

츨

Figura 1. Diâmetro médio geométrico (DMG), Diâmetro médio ponderado (DMP), Índice de Estabilidade de Agregados (IEA) e Índice de Floculação (IF) para os diferentes locais e profundidades estudados. 
FEITOSA, J. R. et al.

Quadro 1. Parâmetros de qualidade do solo avaliados quanto ao número de operações para cada implemento.

\begin{tabular}{|c|c|c|c|}
\hline \multirow{2}{*}{ Implementos } & \multirow{2}{*}{ Variáveis $^{1}$} & $\mathrm{Pt}$ & IF \\
\hline & & \multicolumn{2}{|l|}{$\mathrm{m}^{3} \mathrm{~m}^{-3}$} \\
\hline \multicolumn{4}{|c|}{$\mathrm{N}^{\mathrm{o}}$ de operações } \\
\hline \multirow{4}{*}{ Escarificador } & Uma & $0,431 \mathrm{a}$ & $0,943 \mathrm{~b}$ \\
\hline & Duas & $0,436 \mathrm{a}$ & $0,954 \mathrm{a}$ \\
\hline & Três & 0,438 a & $0,939 \mathrm{~b}$ \\
\hline & $\mathrm{CV}(\%)$ & 4,02 & 0,86 \\
\hline \multirow{4}{*}{ Grade em tandem } & Uma & $0,435 \mathrm{a}$ & $0,935 \mathrm{~b}$ \\
\hline & Duas & $0,434 \mathrm{a}$ & $0,945 \mathrm{a}$ \\
\hline & Três & $0,435 \mathrm{a}$ & $0,949 \mathrm{a}$ \\
\hline & $\mathrm{CV}(\%)$ & 5,18 & 0,86 \\
\hline \multirow{4}{*}{ Grade off-set $(0,56 \mathrm{~m})$} & Uma & $0,425 \mathrm{~b}$ & $0,963 \mathrm{a}$ \\
\hline & Duas & $0,439 a b$ & $0,927 \mathrm{~b}$ \\
\hline & Três & $0,452 \mathrm{a}$ & $0,955 \mathrm{a}$ \\
\hline & $\mathrm{CV}(\%)$ & 4,85 & 1,16 \\
\hline \multirow{4}{*}{ Grade off-set $(0,61 \mathrm{~m})$} & Uma & $0,437 \mathrm{a}$ & $0,540 \mathrm{a}$ \\
\hline & Duas & $0,450 \mathrm{a}$ & $0,487 \mathrm{a}$ \\
\hline & Três & $0,447 \mathrm{a}$ & $0,488 \mathrm{a}$ \\
\hline & CV $(\%)$ & 4,81 & 17,08 \\
\hline
\end{tabular}

Médias seguidas de mesma letra na coluna não diferem entre si pelo Teste de Tukey a $5 \%$ de probabilidade. Pt: Porosidade Total; IF: Índice de Floculação.

faz com que seu processo de decomposição seja acelerado, diminuindo cada vez mais a resistência dos agregados (AGUIAR, 2008).

A ocorrência de agregados menores na camada superficial também pode estar associada à textura arenosa em superfície do Argissolo em questão, visto que a referida classe compreende solos que tem como característica principal a presença de horizonte B textural, o qual apresenta significativo incremento de argila em relação aos horizontes suprajacentes E ou A e argila de baixa atividade (EMBRAPA, 2006).

Assis e Lanças (2010), em trabalho com sistemas de preparo em Nitossolo Vermelho distroférrico, verificaram que o preparo do solo apresentou os menores valores de DMP quando comparado a mata nativa. Os autores ainda verificaram que os menores valores ocorreram na camada superficial analisada $(0,00-0,05 \mathrm{~m})$, corroborando com dados deste trabalho; e que alguns valores elevados de DMP em camadas mais profundas podem ocorrer devido ao tráfego de máquinas que levam a formação de "falsos agregados".

Quanto ao índice de estabilidade de agregados (IEA), não foram encontradas diferenças significativas entre os tratamentos e profundidades 
avaliadas (Figura 1). Uma vez que o IEA representa a média da agregação total do solo sem considerar a distribuição por classes de agregados, Castro Filho et al. (1998), afirmaram que quanto maior a quantidade de agregados, $<0,25 \mathrm{~mm}$, menor será o IEA. Para este trabalho o IEA apresentou valores médios em torno de $73 \%$.

Já o índice de floculação (IF) diferiu significativamente entre os sistemas de preparo do solo utilizados nas camadas de 0,0-0,10 m e 0,20-0,30 m (Figura 1), sendo que na primeira os maiores valores foram encontrados para a área sem preparo, a qual apresentou os menores valores entre 0,20-0,30 m. Quando analisadas apenas as profundidades de coleta, os valores de IF diferiram estatisticamente sendo maiores na camada mais superficial do solo.

Silva et al. (2006) verificaram, para um Argissolo Vermelho, que não houve efeito no índice de floculação para os sistemas de preparo na camada superficial, discordando deste experimento. Grugiki et al. (2009), ao avaliarem Argissolo
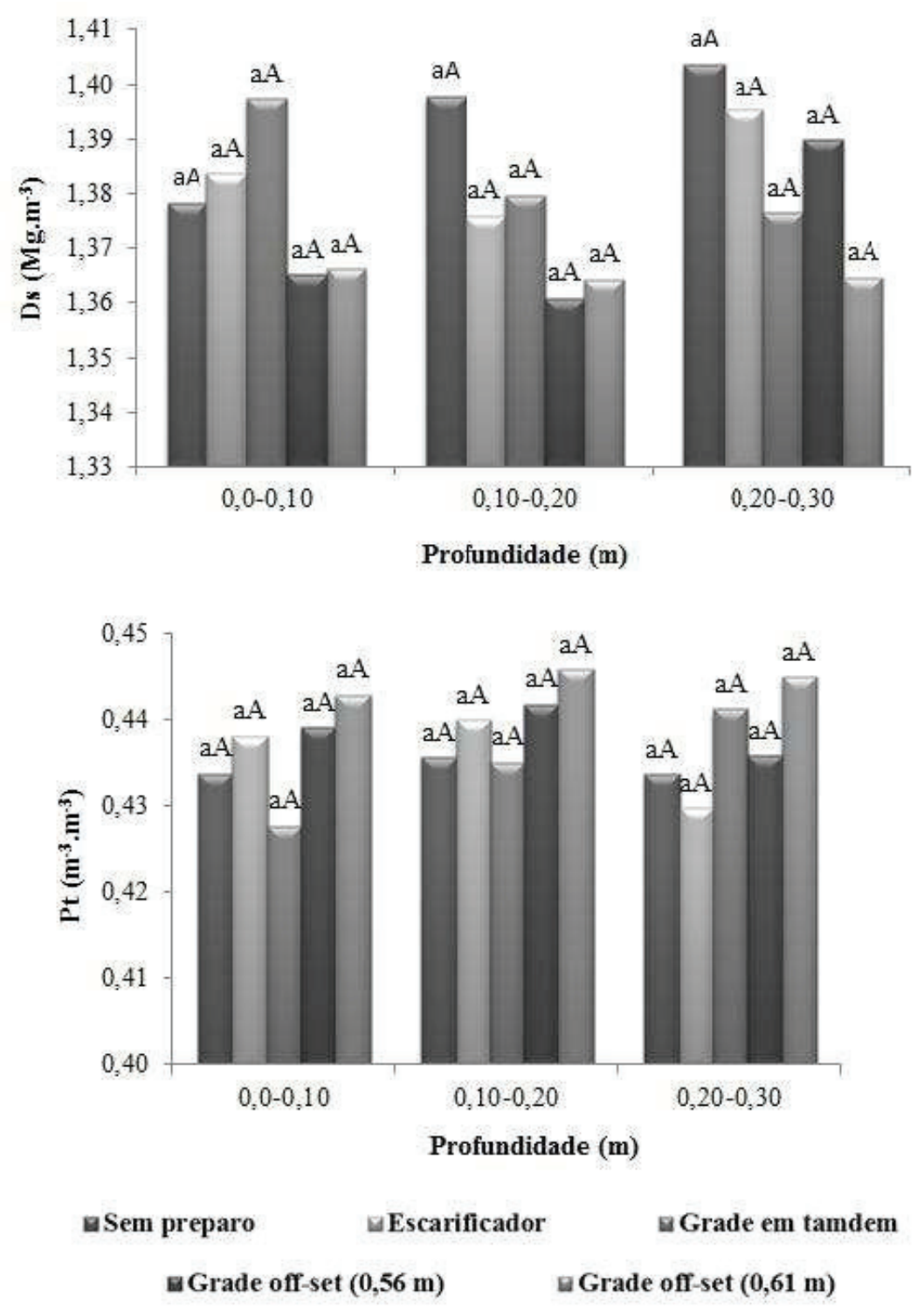

Letras minúsculas: comparação entre os sistemas de preparo em cada profundidade; Letras maiúsculas: comparação entre as profundidades em cada sistema de preparo. Médias seguidas de mesma letra não diferem entre si pelo teste de Turkey a $5 \%$ de probabilidade

Figura 2. Densidade do solo (Ds) e Porosidade (Pt) para os diferentes locais e profundidades estudados. 
Vermelho Amarelo cultivado com mandioca, eucalipto e sorgo, também não encontraram diferenças significativas para o índice de floculação para as profundidades estudadas.

Comparando-se os valores do IF obtidos para cada número de operações (Quadro 1), foram encontradas diferenças significativas para $\mathrm{o}$ escarificador, grade em tandem e grade off-set com discos de $0,56 \mathrm{~m}$, sendo que para os dois primeiros os menores valores foram obtidos para o preparo com uma operação. Para os parâmetros DMG, DMP e IEA, não foram encontradas diferenças significativas entre o número de operações realizado com cada sistema de preparo.

Os valores médios de Ds obtidos nas parcelas submetidas aos sistemas de preparo do solo e número de operações, oscilaram entre 1,36 e $1,40 \mathrm{Mg} \mathrm{m}^{-3}$, considerando-se todas as camadas avaliadas (Figura 2). Não ocorreram diferenças significativas entre os tratamentos, profundidades e número de operação.

Os valores de densidade do Argissolo Amarelo estudado encontram-se dentro dos limites aceitos para um bom desenvolvimento radicular em solos minerais, os quais devem estar entre 1,10 e $1,60 \mathrm{Mg}$ $\mathrm{m}^{-3}$ (KIEHL, 1979). Silva et al. (2002) encontraram paraArgissolo Amarelo de textura média/argilosa da região de Petrolina - $\mathrm{PE}$, valores de 1,46 a $1,50 \mathrm{Mg}$ $\mathrm{m}^{-3}$ nos horizontes $\mathrm{A}$ e $\mathrm{AB}$ e de 1,66 a $1,91 \mathrm{Mg} \mathrm{m}^{-3}$ no horizonte $\mathrm{Bt}$, para áreas sem preparo. Já Cortez et al. (2011) encontraram para Argissolo Amarelo Distrófico típico de textura arenosa localizado em Petrolina-PE e submetido a diferentes sistemas de preparo, valores de densidade que oscilaram entre 1,30 e 1,43 $\mathrm{Mg} \mathrm{m}^{-3}$, considerando-se todas as camadas avaliadas. Segundo os autores, esses valores não são tidos como críticos, uma vez que valores altos de densidade do solo para Argissolos de textura superficial arenosa foram encontrados em outros trabalhos.

A porosidade total $(\mathrm{Pt})$, propriedade que está relacionada diretamente com a densidade do solo, atingiu valores em torno de $0,44 \mathrm{~m}^{3} \mathrm{~m}^{-3}$, não sendo encontradas diferenças significativas entre os resultados obtidos para os diversos tratamentos (Figura 2). Os valores de $\mathrm{Pt}$ encontrados assemelham-se aos obtidos por Cortez et al. (2011), os quais verificaram em Argissolo Amarelo de textura arenosa submetido a diferentes tipos de preparo, no município de Petrolina - PE, valores variando de 0,40 a $0,47 \mathrm{~m}^{3} \mathrm{~m}^{-3}$. Assemelhamse ainda aos obtidos por Silva et al. (2002), que, estudando as características de perfis de Argissolo nos tabuleiros sertanejos de Pernambuco, encontraram valores de porosidade de 0,42 a 0,44 $\mathrm{m}^{3} \mathrm{~m}^{-3}$.

Os valores médios da porosidade total (Figura 2) similares entre os sistemas de preparo do solo e número de operações demonstram que não houve diferença significativa e que os implementos alteraram de forma igualitária os atributos do solo. Cortez et al. (2011) atribuem esse comportamento diferenciado do Argissolo Amarelo à sua textura superficial bastante arenosa, fazendo com que as variações nos atributos porosidade e densidade sejam menores pelo efeito dos sistemas de preparo do solo e número de operações.

\section{CONCLUSÕES}

- Os sistemas de manejo do solo não afetaram de forma significativa o diâmetro médio geométrico, diâmetro médio ponderado e o índice de estabilidade de agregados;

- Os maiores índices de floculação foram encontrados para a camada de 0,0-0,10 m, principalmente na área sem preparo;

- A densidade e a porosidade total do Argissolo Amarelo não foram afetadas pelos sistemas de preparo e o número de operações.

\section{LITERATURA CITADA}

AGUIAR, M.I. Qualidade física do solo em sistemas agroflorestais. 2008. 91f. Dissertação (Mestrado em Solos e Nutrição de Plantas). Universidade Federal de Viçosa, Viçosa, 2008.

AMARAL, F.C.S.; SILVA, E.F. \& MELO. A.S. Caracterização pedológica e estudos de infiltração da água no solo em perímetros irrigados no Vale do São Francisco. Rio de Janeiro: Embrapa Solos, 2006. 104p.

ARAÚJO, E.A.; KER, J.C.; NEVES, J.C.L.; LANI,

\section{REVENG} 456-464p.

ENGENHARIA NA AGRICULTURA, VIÇOSA - MG, V.21 N.5, SETEMBRO / OUTUBRO 2013 
J.L. Qualidade do solo: conceitos, indicadores e avaliação. Revista Brasileira de Tecnologia Aplicada nas Ciências Agrárias. Guarapuava, v.5, n.1, p.187-206, 2012.

ASSIS, L.; LANÇAS, K.P. Agregação de um Nitossolo Vermelho distroférrico sob sistemas de plantio direto, preparo convencional e mata nativa. Revista Engenharia Agrícola, Jaboticabal, v.30, p.58-66, 2010.

BERTOL, I.; ALBUQUERQUE, J.A.; LEITE, D.; AMARAL, A.J.; ZOLDAN JUNIOR, W.A. Propriedades físicas do solo sob preparo convencional e semeadura direta em rotação e sucessão de culturas comparadas às de campo nativo. Revista Brasileira de Ciência do Solo, Viçosa, v.28, p.155-163, 2004.

CASTRO FILHO, C.; MUZILLI, O.; PODANOSCHI, A.L. Estabilidade dos agregados e sua relação com o teor de carbono orgânico num Latossolo Roxo distrófico, em função de sistemas de plantio, rotações de culturas e métodos de preparo de amostras. Revista Brasileira de Ciência do Solo, Viçosa, v.22, p.527-538, 1998.

CORTEZ, J.W.; ALVES. A.D.S.; MOURA, M.R.D.; OLSZEVSKI, N.; NAGAHAMA, H.J. Atributos físicos do Argissolo Amarelo do semiárido nordestino sob sistemas de preparo. Revista Brasileira de Ciência do Solo, Viçosa, v.35, p.1207-1216, 2011.

EMBRAPA - EMPRESA BRASILEIRA DE PESQUISA AGROPECUÁRIA. Manual de métodos de análise do solo. Rio de Janeiro: Centro Nacional de Pesquisas de Solos, 1997. 212p.

EMBRAPA - EMPRESA BRASILEIRA DE PESQUISA AGROPECUÁRIA. Sistema Brasileiro de Classificação de Solos. Brasília, 2006. 306p.

GRUGIKI, M.A.; GODINHO. T.O.; VIEIRA, M.V.M.; SANTOS, C.L.; PASSOS， R.R.; RANGEL, O.J.P. Argila dispersa em água e grau de floculação de um Argissolo Vermelho Amarelo sob diferentes coberturas vegetais. In: ENCONTRO LATINO AMERICANO DE INICIAÇÃO CIENTÍFICA, 13, 2009. São José dos Campos. Anais eletrônicos... São José dos Campos: Universidade do Vale da Paraíba, 2009.

KEMPER, W.D.; CHEPIL, W.S. Size distribution of aggregate. In: BLACK, C.A. (Ed.). Methods of soil analysis. Part. 1. Madison, American Society of Agronomy, p. 499-509, 1965.

KIEHL, E.J. Manual de Edafologia: relações solo-planta. São Paulo: Agronômica Ceres, 1979. $263 p$.

MAZURANA, M.; LEVIEN, R.; MÜLLER, J.; CONTE, O. Sistemas de preparo de solo; alterações na estrutura do solo e rendimento das culturas. Revista Brasileira de Ciência do Solo, Viçosa, v.35, p.1197 - 1206, 2011.

MELO, V.F.; MOURA, R; TOLEDO, F.H.; LIMA, V.C. \& GHIDIN, A.A. Morfologia de agregados de Latossolos Bruno e Vermelho do estado do Paraná, avaliada por imagens obtidas em scanner. Revista Brasileira de Ciência do Solo, Viçosa, v.32, p.8599, 2008.

MORAES, A.G.L.; LOSS, A.; PEREIRA, M.G.; ANJOS, L.H.C. \& SILVA, E.M.R. Distribuição dos agregados e diâmetro médio ponderado em diferentes sistemas de produção orgânica. Resumos do VI CBA e II CLAA, Revista Brasileira de Agroecologia, Porto Alegre, v.4, p.1302-1305, 2009.

MENDES, F.G.; MELLONI, E.G.P.; MELLONI, R. Aplicação de atributos físicos do solo no estudo da qualidade de áreas impactadas, em Itajubá/MG. Cerne, Lavras, v.12, n.3, p.211-220, 2006.

PEDROTTI, A.; MÉLLO JÚNIOR, A.V. Avanços em Ciência do Solo: A Física do solo na Produção Agrícola e Qualidade Ambiental. São Cristovão: Editora UFS, Acaraju: Fapitec, 2009. 212p.

SILVA, F.A.S. \& AZEVEDO, C.A.V. Versão do Programa computacional Assistat para o sistema operacional Windows. Revista Brasileira de 
Produtos Agroindustriais, Campina Grande, v.4, p.71-78, 2002.

SILVA, I.F.; MIELNICZUK, J. Sistemas de cultivo e características do solo afetando a estabilidade de agregados. Revista Brasileira de Ciência do Solo, Viçosa, v.22, p.311-317, 1998.

SILVA, M.S.L.; KLANT, E.; CAVALCANTI, A.C.; KROTH, P.L. Adensamento subsuperficial em solos do semiárido: processos geológicos e/ou pedogenéticos. Revista Brasileira de Engenharia Agrícola e Ambiental, Campina Grande, v.6, p.314-320, 2002.
SILVA, M.A.S.; MAFRA,A.L.;ALBUQUERQUE, J.A.; ROSA, J.D.; BAYER, C.; MIELNICZUK, J. Propriedades físicas e teor de carbono orgânico de um argissolo vermelho sob distintos sistemas de uso e manejo. Revista Brasileira de Ciência do Solo, Viçosa, v.30, p.329-337, 2006.

TORMENA, C.A.; BARBOSA, M.C.; COSTA, A.C.S.; GONÇALVES, C.A. Densidade, porosidade e resistência à penetração em Latossolo cultivado sob diferentes sistemas de preparo do solo. Revista Scientia Agrícola, Piracicaba, v.59, n. 4, p. 795-801, 2002. 